\title{
Erratum
}

\section{On the anomalies in Lorentz-breaking theories}

\author{
[Int. J. Mod. Phys. A, Vol. 31, No. 12, 1650063 (2016)] \\ A. P. Baeta Scarpelli \\ Centro Federal de Educação Tecnológica - MG, \\ Avenida Amazonas, 7675 - 30510-000 - Nova Gameleira - Belo Horizonte - MG, Brazil \\ Setor Técnico-Científico - Departamento de Polícia Federal, \\ Rua Nascimento Gurgel, 30 - Gutierrez - Belo Horizonte - MG, Brazil \\ scarpelli.apbs@dpf.gov.br \\ T. Mariz \\ Instituto de Física, Universidade Federal de Alagoas, \\ 57072-270, Maceió, Alagoas, Brazil \\ tmariz@fis.ufal.br \\ J. R. Nascimento* and A. Yu. Petrov ${ }^{\dagger}$ \\ Departamento de Física, Universidade Federal da Paraíba, \\ Caixa Postal 5008, 58051-970, João Pessoa, Paraíba, Brazil \\ *jroberto@fisica.ufpb.br
}

Received 13 September 2017

Published 20 September 2017

1. The third line above Eq. (14) should be read as:

"The variation of the measure will be obtained by the replacement of $\tilde{F}_{m n}$ by $\bar{F}_{m n}=\bar{\partial}_{m} \bar{A}_{n}-\bar{\partial}_{n} \bar{A}_{m}(\ldots)$."

2. Equation (20) should be read as:

$$
\begin{aligned}
\delta \mathcal{L}= & C\left\{e^{2} \epsilon^{a b c d} b_{a} A_{b} \partial_{c} A_{d}+2 e g_{2}\left[b^{2} F_{c d} F^{c d}-2\left(b_{c} F^{c d}\right)^{2}\right]\right. \\
& \left.+2 g_{2}^{2}\left(b^{2} F^{c d}+b_{a} b^{c} F^{d a}\right) \epsilon_{d m n p} b^{m} \partial_{c} F^{n p}\right\} .
\end{aligned}
$$


A. P. Baeta Scarpelli et al.

3. In the third line above Eq. (21) we should have: "(..) and a modified gauge field, $\bar{A}_{m}=\tilde{A}_{m}+c_{m n} \tilde{A}^{n}(\ldots) . "$

None of the conclusions of our paper are changed.

\section{Acknowledgment}

The work has been partially supported by CNPq. 